\title{
Measurement and Analysis of Vehicle Noise on the Yamanashi Maglev Test Line
}

\section{Toshio HIROTA}

General Manager, Vehicle Engineering Div., Maglev Systems Development Dept.

\section{Yasuo ZENDA}

Chief Engineer, Aerodynamic and Noise Reduction G., Environmental Preservation \& Disaster Prevention Technology Development Div., Technological Development Dept.

\section{Shiro HOSAKA}

Senior Engineer, Linear Express Development Div., Central Japan Railway Company

\begin{abstract}
We set three microphones, each with a parabolic reflector, to measure vehicle noise on the Yamanashi Maglev Test Line. Using a personal computer, we analyze the noise from the surface of the car. In this paper, we explain the noise measuring system used for this study and data processing, focusing on the calculation of sound-levels, analysis of time-dependent signals and visualization of the noise. We started to measure the noise in April 1999 to collect the data required to reduce the noise level. There is no noise from the car windows as has been expected, since we have designed the window to be smooth and even on the outer surfaces, while high-frequency noise from around the trucks and doors is recognized at $500 \mathrm{~km} / \mathrm{h}$. The noise of gas turbine is at low levels.
\end{abstract}

Keywords : Maglev, Yamanashi Maglev Test Line, noise, digital signal processing, parabolic microphone

\section{Introduction}

The vehicle running test on the Yamanashi Maglev Test Line began in April 1997. The running test continued with no major problems. A manned vehicle running speed of $552 \mathrm{~km} / \mathrm{h}$ was achieved in April 1999. We are evaluating the applicability of Maglev system to commercially operated lines through experimentation. For this purpose, the vehicle noise must be reduced further.

We use devices that combine a parabolic reflector and a microphone as shown in Fig. 1 for the Yamanashi Maglev Test Line to analyze the vehicle noise efficiently. We call

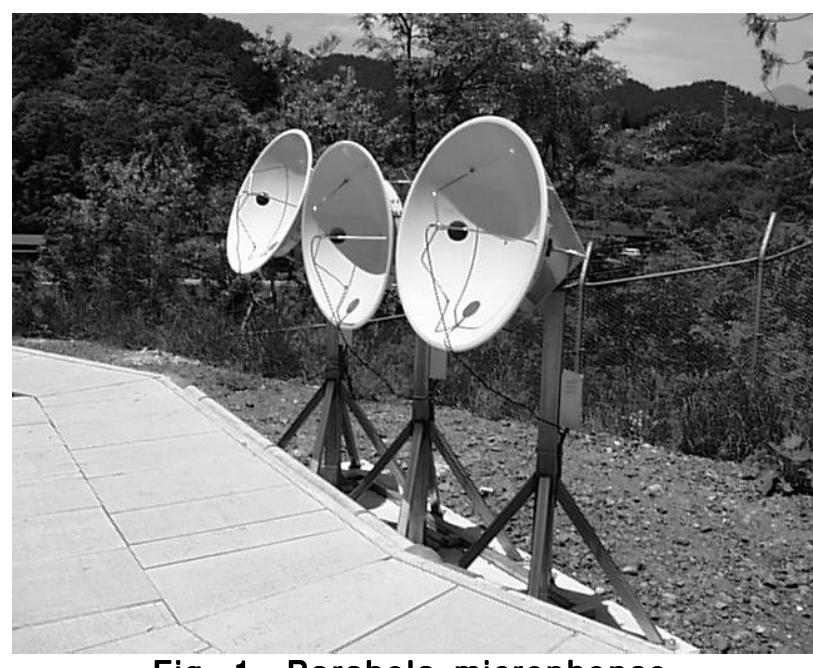

Fig. 1 Parabola microphones these devices parabolic microphones. As shown in Fig.4 the noise from the vehicle side is measured at the upper part, lower part and middle part with the parabolic microphone. We developed a computer program to analyze the noise measured by the parabolic microphones. This paper explains an outline of noise data processing.

\section{Outline of the parabolic microphone}

The parabolic microphone has been used to measure the noise of Shinkansen. The reflector of the parabolic microphone has a form to cut the rotating parabolic face

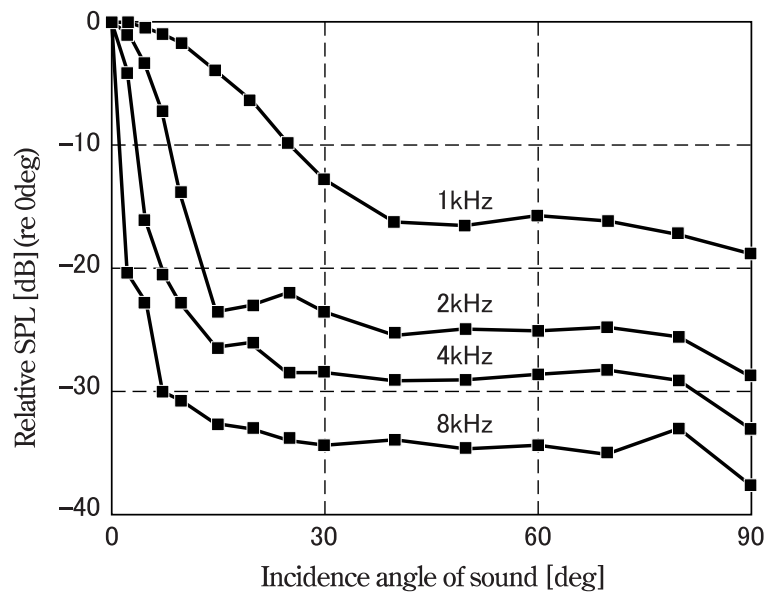

Fig 2. Characteristics of parabola microphone 


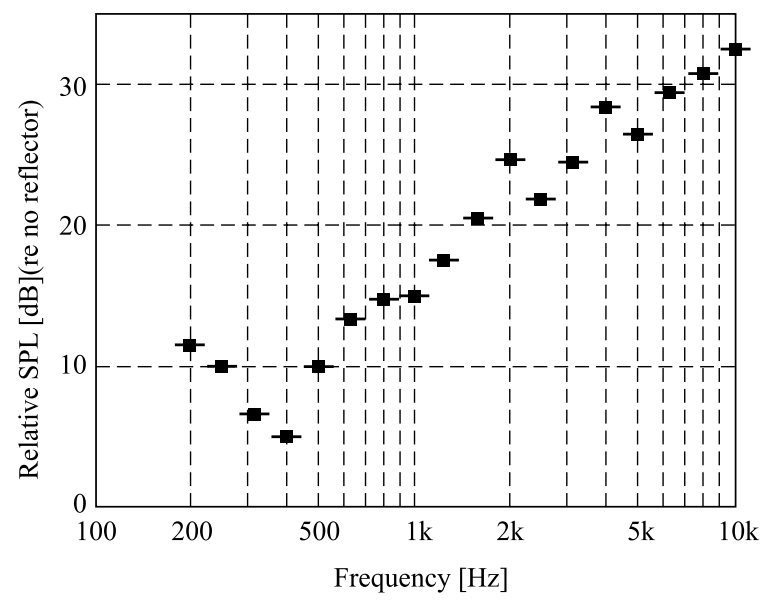

Fig. 3 Gain characteristics of parabola microphone

in the plane at the right angle to the axis that passes the focus point. The diameter of the reflector of the parabolic microphone is $1 \mathrm{~m}$. It has a microphone at the focus. The characteristics of directivity of this parabolic microphone as a parameter of frequency are shown in Fig.2. Also, the characteristics of the gain are shown as a parameter of frequency in Fig. 3. As the frequency of noise becomes high, the directivity becomes sharp, and the gain becomes high. The measurement of the noise by the parabolic microphone that has such characteristics differs from the measurement of environmental noise. High frequency components are included in the noise from the vehicle surface. The characteristics of this parabolic microphone are adequate for investigating the noise that occurs from the vehicle surface.

As shown in Fig.4, the three parabolic microphones measure the noise from the upper part, lower part and center part of the vehicle side that has a height of $3 \mathrm{~m}$. These parabolic microphones we placed at intervals of $1.2 \mathrm{~m}$. The search coil of the air core is attached to the outside of the guide-way. A voltage occurs in these search coils by the fluctuation of the magnetic field as the superconducting magnets move. We can understand the relative distance between the vehicle and parabolic micro-

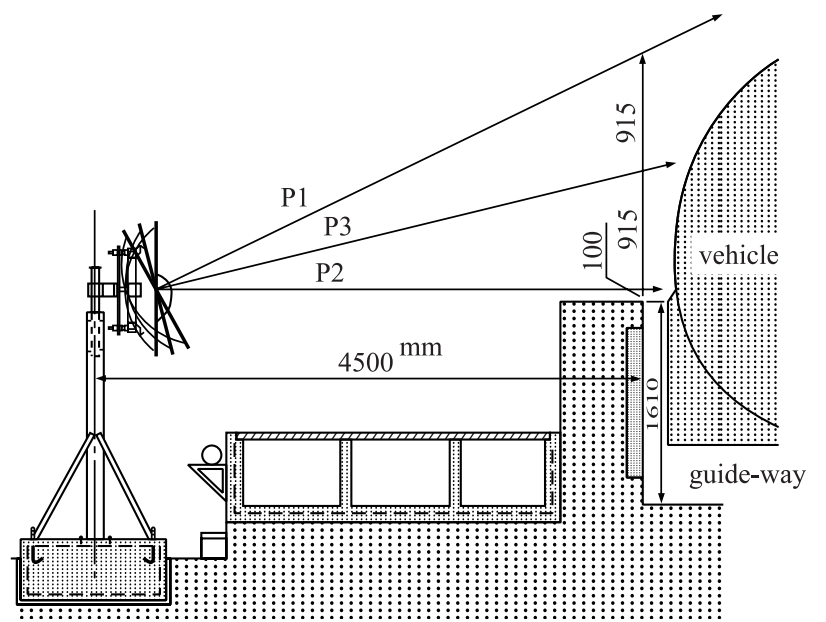

Fig. 4 Parabola microphone and vehicle phones, speed and running direction of the vehicle by the voltage in the search coil. The difference of location of the parabolic microphone and the difference of the distance from the surface of the vehicle were counted when they were output as a chart.

\section{Digital Signal Processing}

The data of noise is recorded in the media of CD-R. The program runs on a personal computer.

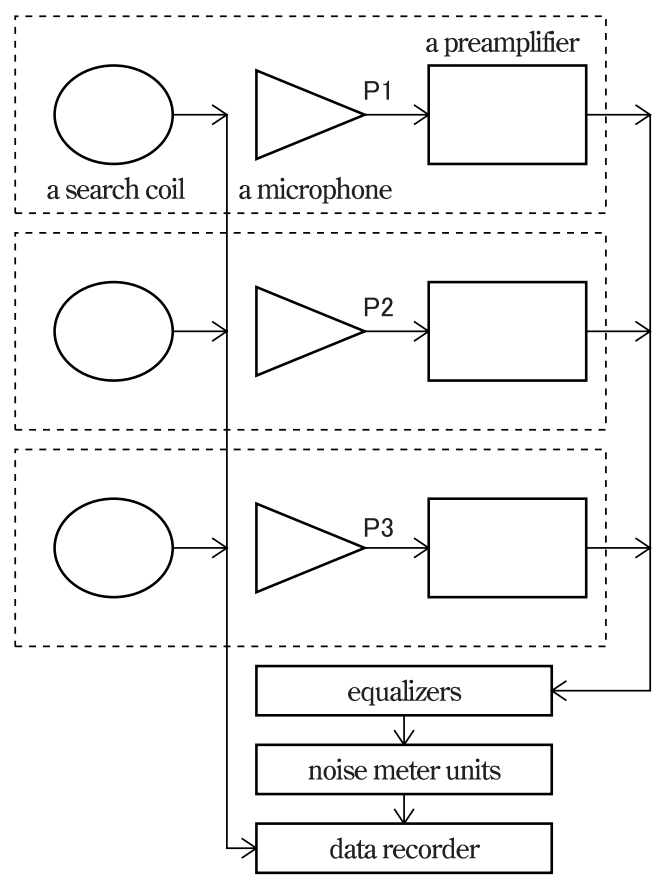

Fig. 5 Measurement system

\subsection{Sound-levels and RMS}

Generally, the sound level value in the environmental standards is measured with the standard instruments. In this paper, however, the sound level value is calculated from the sound pressure value without using the standard instruments. The method to calculate the sound level value is shown below.

By using the time constant $T$ and sound pressure $p(t)$, the sound level value $L(t)$ is defined as:

$$
L(t)=10 \log _{10}\left[\frac{1}{T} \int_{-\infty}^{t}\left\{\frac{p(\tau)}{p_{0}}\right\}^{2} e^{-\frac{t-\tau}{T}} d \tau\right] .
$$

We define the following function $S(t)$ by using the sampling interval $\Delta t$.

$$
S(t)=\frac{1}{\Delta t} \int_{-\infty}^{t} p^{2}(\tau) e^{-\frac{t-\tau}{T}} d \tau
$$

$S(t)$ is calculated repeatedly.

$$
S(t) \cong S(t-\Delta t) e^{-\frac{\Delta t}{T}}+p^{2}(t)
$$

By using this formula the sound level value $L(t)$ is calculated as: 


$$
L(t)=10 \log _{10}(S(t))-10 \log _{10}\left(\frac{T}{\Delta t}\right)-20 \log _{10} p_{0}
$$

The second and third terms are constant. The sound level value $L(t)$ is calculated by the following algorithm.

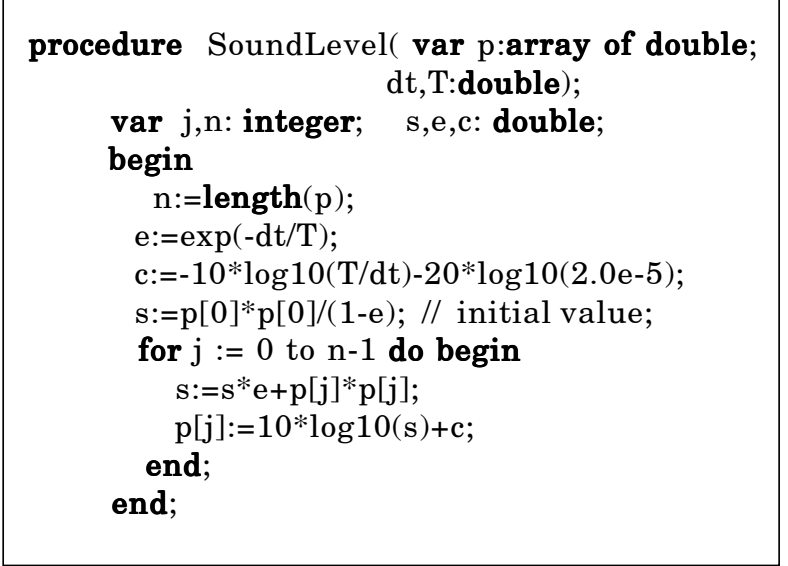

The initial value of $S(t)$ is unknown. We suppose that $S(-\Delta t)$ equals $S(0)$.

$$
S(-\Delta t)=S(0)=S(-\Delta t) * e+p[0] * p[0]
$$

and we obtain

$$
S(0)=p[0] * p[0] /(1-e) .
$$

If the time constant is large, the sound level value continues long. The sound level corresponding to the vehicle surface depends on the running direction. RMS (root mean square) of sound pressure does not depend on the running direction. RMS is calculated by the following equation.

$$
P_{r m s}(t)=\sqrt{\frac{1}{2 M+1} \sum_{k=-M}^{M} p^{2}(t+k \Delta t)} .
$$

The integration time $(2 M+1) \Delta t$ corresponds to the time constant $\mathrm{T}$.

\section{$3.2 \quad$ Filters}

The components of high frequency are included in the sound that occurs from the angular part of the vehicle surface. Furthermore, the movement of pressure wave on the vehicle periphery is observed as a low frequency sound pressure. We can observe the sound source of the vehicle surface in detail by using a high-pass filter to remove the components of low frequency of sound pressure. We are using a digital filter that can freely change the characteristics of frequency after measurement. We use the digital filter called the Kaiser filter. This corresponds to off-line processing with the fine phase characteristics, while it requires time to calculate. The digital filters of the lowpass filter, band-pass filter and high-pass filter are calculated by the same method. Therefore, filters of any type can be used in our system.

\subsection{Time-Dependent Signal}

The frequency and strength of noise in passenger cabins change with the speed. Furthermore, the frequency of sound changes by the Doppler effect even at the same vehicle speed at wayside. It is important to know the timedependent frequency and the source of noise precisely. Many methods are being studied regarding the analysis of time-dependent signal. The Short-time Fourier Transform, that divides data in short periods and calculates the components of frequency, is one example of these methods. The unnecessary components of frequency increase when a rectangle window function is used. Several kinds of window functions are used to decrease unnecessary components of frequency.

The product of the dispersion of time and frequency is not smaller than a particular value. In other words, it is not possible to know the time and the frequency of the sound at the same time.

The Short-time Fourier Transform as the window function of Gaussian function is called Gabor Transform. When Gaussian function is used as a window function, the dispersion of time and frequency becomes the minimum. Gabor Transform is defined by using the following function :

$$
\begin{gathered}
g_{f, \tau}(t)=A e^{-\frac{(t-\tau)^{2}}{\sigma^{2}}} e^{j 2 \pi f t} . \\
G(f, \tau)=\int_{-\infty}^{\infty} g_{f, \tau}^{*}(t) x(t) d t .
\end{gathered}
$$

The asterisk denotes the conjugate complex value.

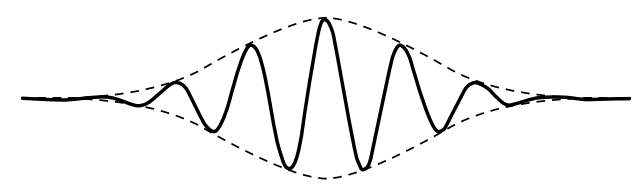

1) Real part (cosine type)

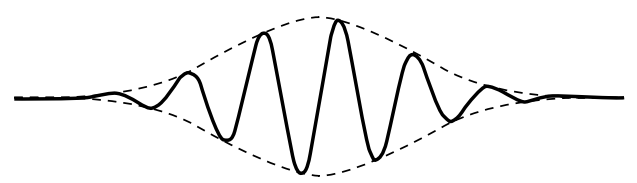

2) Imaginary part (sine type)

\section{Fig. 6 Wavelet (Gabor function)}

The Wavelet Transform which uses a small wave that has a limited time width is proposed to improve the Fourier Transform which uses sine wave that has an infinite time width. The basis is made by using variables a and $b$ for the shift and dilation on the time axis, based on a wavelet.

$$
\varphi_{a, b}(t)=\frac{1}{\sqrt{a}} \varphi\left(\frac{t-b}{a}\right) .
$$

The signal $x(t)$ is transformed by using this basis.

$$
W(a, b)=\int_{-\infty}^{\infty} \varphi_{a, b}^{*}(t) x(t) d t
$$

The integration range of expression has an infinite time width. In the Wavelet Transform, however, the integration range is limited, because the wavelet function value is zero at the infinite time.

The wavelet is studied prosperously regarding its form and nature. The application of the wavelet to the 
compression of images and the noise reduction is reported. If the frequency becomes higher in the Wavelet Transform, the integral time width becomes shorter. And the dispersion in time becomes small. This is a good characteristic to know the source of noise by the observation in a short time. We analyze the vehicle noise by using Wavelet Transform that uses the Gabor function.

When Gabor function is used:

$$
\varphi_{\sigma}(t)=\frac{1}{\sigma \sqrt{\pi}} e^{-\frac{t^{2}}{\sigma^{2}}} e^{j t}
$$

And using the Fourier Transform, we obtain

$$
X(\omega)=\int_{-\infty}^{\infty} x(t) e^{-j \omega t} d t .
$$

We get the following equation.

$$
W(a, b)=\frac{\sqrt{a}}{2 \pi} \int_{-\infty}^{\infty} X(\omega) e^{-\frac{\sigma^{2}}{4}(1-a \omega)^{2}} e^{j b \omega} d \omega .
$$

This expression shows the characteristic of the bandpass filter that has Gaussian distribution. The frequency that has a decrease which is a half of the decrease of central frequency, is as follows.

$$
f=\frac{1}{2 \pi a}\left(1 \pm \frac{\sqrt{2 \ln 2}}{\sigma}\right)
$$

This expression shows that the band width of a bandpass filter is decided by $\sigma$. The band width at $\sigma=10$ is almost equal to that of a $1 / 3$ octave band filter.

The Integral Wavelet Transform is calculated by digital computing. Because the wavelet that is shown in Fig. 6 has a limited length, it is sufficient to calculate in a limited time.

$$
G(a, b)=\frac{1}{\sqrt{a}} W(a, b)
$$

Putting $a=k \Delta t, b=m \Delta t, t=n \Delta t, x(n \Delta t)=x_{n}$, we obtain

$$
G(k, m)=\frac{1}{k} \sum_{l=-\infty}^{\infty} \varphi_{\sigma}^{*}\left(\frac{l}{k}\right) x_{m+l}
$$

Using a complex number array:

$$
C_{k, l}=\varphi_{\sigma}\left(\frac{l}{k}\right)
$$

We obtain

$$
\begin{gathered}
G(k, m)=\frac{1}{k} \sum_{l=-k N}^{k N} C_{k, l}^{*} x_{m+l}, \\
\text { where }\left|\varphi_{\sigma}(Z)\right| \cong 0 \text {, for all } Z \geq N .
\end{gathered}
$$

Using a sampling frequency $f_{s}$, and the center frequency $f_{0}$ of the band-pass filter, we get the normalized scale.

$$
k_{0}=\frac{f_{s}}{2 \pi f_{0}} .
$$

The components of frequency can be calculated by digital computing with good dispersion of time and frequency.

\subsection{Visualization}

We believe that speed does not change because the observation time of the parabolic microphones is a half second when the vehicle that has a length of $70 \mathrm{~m}$ runs at $500 \mathrm{~km} / \mathrm{h}$. Therefore, the vehicle position is proportional to the observation time. It is necessary to understand the source of noise to know the frequency and strength of sound corresponding to the position of the vehicle. The frequency and strength of sound is displayed on the basis of the position of the vehicle in our system. We use two methods to display the data of the noise.

The strength of noise is calculated at regular intervals from the data of three parabolic microphones in the first method. The distribution of strength is displayed in these data in the side view of the vehicle by using contour lines. The noise level of the locations that are not directly measured are calculated by linear interpolation.

The components of the frequency that was calculated by using Gabor function is displayed in the second method. The noise has the three elements of position, frequency and strength. The strength of the noise is expressed by the color or brightness to display three-dimensional data in the two-dimensional display.

\section{Results}

The result of a 5 -car train set that ran at $450 \mathrm{~km} / \mathrm{h}$ is shown in Fig.7. The vehicle is a tail end car. The bandpass filter of $1.4 \sim 2.8 \mathrm{kHz}$ is used. The voltage of the search coil is shown in 1). The dotted line shows the position of center and edge of truck. The contour lines in the side view of the vehicle and noise level are shown in 2 ). The contour lines by the root mean square of sound pressure are shown in 3). The result of the frequency analysis of the data by the upper part $\mathrm{P} 1$ of the vehicle side is shown in 4).

A gas turbine and generator is installed to this vehicle as the auxiliary power supply source on the car. The serious noise by the gas turbine did not occur. We used a smooth structure of the window of the vehicle side. The window is not observed as the source of noise. The noise occurred at the central door of the vehicle side according to the contour lines of noise level and the root mean square of sound pressure. We think this caused by the opening and difference in level of the door. Noise occurs at the part where the section of the vehicle changes and the part with a difference in level. Especially, noise occurs from the periphery of trucks and the part of coupling. Because the band-pass filter is working, only the data of the band is displayed. As shown in notes, the deep color part is a high level component of the noise.

The component of the frequency at the upper part of the vehicle side corresponds to the result of the upper part that was shown with the contour lines.

\section{Conclusions}

The parabolic microphones operated from April 1999. We are analyzing a lot of data. By assessing the source of noise precisely by using signal analysis, we will reduce the noise. The application of the analysis method of the frequency that is described in this paper is not only for 


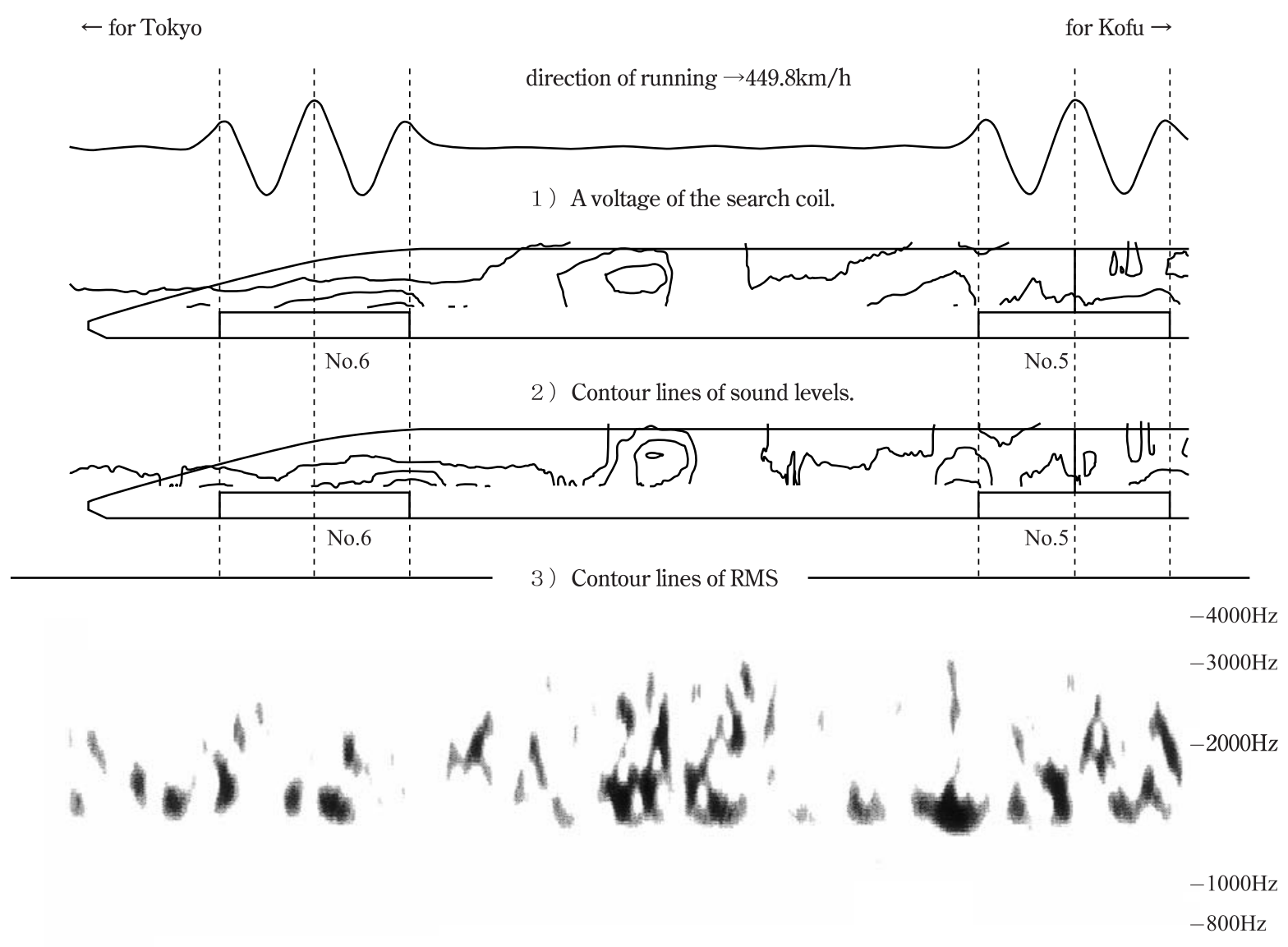

4) Spectrogram

$62 \mathrm{~dB}$

$66 \mathrm{~dB}$

$69.75 \mathrm{~dB}$

Fig. 7 The result of a 5 -cars train set $(450 \mathrm{~km} / \mathrm{h})$

noise analysis. We also use it for the vibration analysis of superconducting magnets. The running speed can be calculated precisely from the vibration frequency of superconducting magnets.

The experiment and development in the Yamanashi Maglev Test Line are subsidized by the Ministry of Transport of Japan.

\section{Acknowledgment}

The parabolic microphone is a device that has been used in the aerodynamic and noise reduction group. We constructed a program based on their work.

We thank everybody of the aerodynamic and noise reduction group and Yamanashi Maglev Test Center who cooperated with this research.

\section{References}

1) Y. Zenda,Y. Simizu,K. Nagakura: "Challenge to Shinkansen noise", RRR, 52-5, pp.5-8,1995

2) Y. Zenda, T. Kitagawa: "Noise a la carte", RRR, 5411,pp.22-25,1997

3) Sophocles J.Orfanidis: Introduction to Signal Processing, pp.551-567, Prentice Hall International, Inc.

4) Sirou Sirai: Signal Processing, OumuSha, 1998

5) Hirosi. Toda: "Theory and practice of the latest signal analysis technology", C Magazine,10-12, pp.2862,1998 